\title{
Mean platelet volume and cardiac-surgery-associated acute kidney injury: a retrospective study
}

\section{Volume plaquettaire moyen et insuffisance rénale aiguë associée à la chirurgie cardiaque : une étude rétrospective}

\author{
Abinaya Ramakrishnan $\cdot$ Cynthia Zheng, BA $\cdot$ Manuel L. Fontes, MD • \\ Frederic W. Lombard, MBChB, FANZCA • Austin A. Woolard, MD • Yaping Shi, MS • \\ Matthew S. Shotwell, PhD • Frederic T. Billings 4th, MD • Mias Pretorius, MBChB, \\ MSCI · Jonathan P. Wanderer, MD, MPhil, FASA, FAMIA - Rushikesh Vyas, MD • \\ Tarek S. Absi, MD • Ashish S. Shah, MD • Miklos D. Kertai, MD, PhD
}

Received: 8 May 2020/Revised: 26 June 2020/ Accepted: 1 July 2020/Published online: 15 September 2020

(c) Canadian Anesthesiologists' Society 2020

\begin{abstract}
Purpose Increased mean platelet volume (MPV) may indicate platelet activation, platelet aggregation, and a resulting prothrombotic state. Such changes in the postoperative period have been associated with organ injury and adverse outcomes. We hypothesized that changes in MPV after cardiac surgery are associated with both a higher risk of acute kidney injury (AKI) and mortality.
\end{abstract}

Electronic supplementary material The online version of this article (https://doi.org/10.1007/s12630-020-01811-4) contains supplementary material, which is available to authorized users.

A. Ramakrishnan

Vanderbilt University, Nashville, TN, USA

C. Zheng, BA

Rutgers Robert Wood Johnson Medical School, New Brunswick, NJ, USA

M. L. Fontes, MD

Yale School of Medicine, New Haven, CT, USA

F. W. Lombard, MBChB, FANZCA - A. A. Woolard, MD ·

F. T. Billings 4th, MD - M. Pretorius, MBChB, MSCI

Department of Anesthesiology, Vanderbilt University Medical

Center, Nashville, TN, USA

Y. Shi, MS · M. S. Shotwell, PhD

Department of Biostatistics, Vanderbilt University Medical

Center, Nashville, TN, USA

J. P. Wanderer, MD, MPhil, FASA, FAMIA

Department of Anesthesiology, Vanderbilt University Medical

Center, Nashville, TN, USA
Methods In this retrospective study, we evaluated consecutive patients undergoing adult cardiac surgery patients between 12 December 2011 and 5 June 2018. The change in MPV was derived by calculating the difference between the baseline MPV before surgery and the average postoperative MPV just prior to the occurrence of AKI. We defined postoperative AKI according to Kidney Disease: Improving Global Outcomes Clinical Practice Guideline for Acute Kidney Injury as either $a \geq 50 \%$ increase in serum creatinine in the first ten postoperative days, or an

Department of Biomedical Informatics, Vanderbilt University Medical Center, Nashville, TN, USA

R. Vyas, MD - T. S. Absi, MD - A. S. Shah, MD

Department of Cardiac Surgery, Vanderbilt University Medical Center, Nashville, TN, USA

M. D. Kertai, MD, PhD ( $)$

Department of Anesthesiology, Vanderbilt University Medical Center, Nashville, TN, USA

e-mail: miklos.kertai@vumc.org

Perioperative Precision Medicine Program, Division of Adult Cardiothoracic Anesthesiology, Department of Anesthesiology, Vanderbilt University Medical Center, 1211 21st Avenue South, Medical Arts Building, Office 526, Nashville, TN 37212, USA 
increase of $\geq 0.3 \mathrm{mg} \cdot \mathrm{dL}^{-1}$ during any 48 - $\mathrm{hr}$ window across the ten-day postoperative period. Multivariable logistic regression analysis was used to examine the association between MPV change and postoperative AKI and mortality.

Results Of the 4,204 patients studied, 1,373 (32.7\%) developed postoperative AKI, including 83 (2.0\%) and 38 (0.9\%) who developed stages II and III AKI, respectively. Compared with patients who had an increase in median postoperative MPV of 0.2 femtolitre ( $\mathrm{L} L)$, those with an increase of $0.8 \mathrm{fL}$ had an $80 \%$ increase in the odds of developing AKI (adjusted odds ratio [aOR], 1.80; 95\% confidence interval [CI],1.36 to 2.38; $P<0.001)$ and were almost twice as likely to progress to a higher severity AKI (aOR, 1.66; $95 \%$ CI, 1.28 to 2.16; $P<0.001)$. Change in MPV was not associated with mortality (aOR,1.32; $95 \%$ CI, 0.92 to $1.89 ; P=0.14$ ).

Conclusion Increased MPV change in the postoperative period was associated with both increased risk and severity of AKI, but not mortality.

\section{Résumé}

Objectif Un volume plaquettaire moyen (VPM) augmenté peut être indicatif d'une activation plaquettaire, d'une agrégation plaquettaire, et de l'état prothrombotique qui en résulte. De tels changements en période postopératoire ont été associés à des lésions aux organes et à des devenirs défavorables. Nous avons émis l'hypothèse que des changements du VPM après une chirurgie cardiaque seraient associés à un risque plus élevé d'insuffisance rénale aiguë et de mortalité.

Méthode Dans cette étude rétrospective, nous avons évalué des patients adultes consécutifs subissant une chirurgie cardiaque entre le 12 décembre 2011 et le 5 juin 2018. Le changement de VPM a été dérivé en calculant la différence entre le VPM de base avant la chirurgie et le VPM postopératoire moyen juste avant la survenue de l'IRA. Nous avons défini une IRA postopératoire sur la base des Directives Kidney Disease: Improving Global Outcomes Clinical Practice Guideline for Acute Kidney Injury (Les maladies rénales: Guide d'exercice clinique pour améliorer les devenirs globaux pour l'insuffisance rénale aiguë) en tant qu'une augmentation $\geq 50 \%$ de la créatine sérique au cours des dix premiers jours postopératoires, ou une augmentation de $\geq 0,3 \mathrm{mg} \cdot \mathrm{dL}^{-1}$ pendant toute fenêtre de $48 \mathrm{~h}$ au cours des dix premiers jours postopératoires. Une analyse multivariée de régression logistique a été utilisée pour examiner l'association entre le changement de VPM et l'IRA postopératoire et la mortalité.

Résultats Parmi les 4204 patients à l'étude, 1373 (32,7 $\%)$ ont souffert d'IRA postopératoire, y compris 83 (2,0\%) et 38 (0,9\%) qui ont développé des IRA de stade II et III, respectivement. Par rapport aux patients ayant manifesté une augmentation du VPM postopératoire médian de 0,2 femtolitre ( $f L)$, ceux affichant une augmentation de 0,8 fL ont démontré une augmentation de $80 \%$ de la probabilité d'IRA (rapport de cotes ajusté [RCA], 1,80; intervalle de confiance [IC] $95 \%, 1,36$ à 2,38; $P<0,001)$ et couraient un risque pratiquement deux fois plus élevé de voir leur IRA progresser à un stade plus grave (RCA, 1,66; IC $95 \%$, 1,28 à 2,16; $P<0,001)$. Les changements de VPM n'étaient pas associés à la mortalité (RCA, 1,32; IC $95 \%$, 0,92 à 1,89; $P=0,14$ ).

Conclusion Une augmentation accrue du VPM en période postopératoire a été associée à un risque et une gravité accrus d'IRA, mais pas à la mortalité.

Keywords acute kidney injury · cardiac surgery · platelet count $\cdot$ mean platelet volume $\cdot$ mortality

\section{Introduction}

Of the 235,000 cardiac surgical procedures performed annually in the United States, ${ }^{1}$ approximately $30-50 \%$ of patients develop postoperative acute kidney injury (AKI), and 1-5\% develop severe kidney injury requiring renal replacement therapy. ${ }^{2,3}$ The presence and severity of postoperative AKI have been shown to increase both hospital length of stay and rates of in-hospital mortality. Indeed, cardiac surgery patients with postoperative AKI are more likely to require intensive and supportive care and hospital readmissions, and to experience a decreased quality of life and long-term survival, compared with those without postoperative AKI. ${ }^{3-5}$

The etiology of postoperative AKI after cardiac surgery is complex and multifactorial. ${ }^{2,4,5}$ It involves patientrelated risk factors including advanced age, obesity, chronic kidney disease, diabetes mellitus, poor left ventricular function, and hypertension, as well as surgery-related factors such as ischemia-reperfusion injury, inflammatory responses, procoagulant states, and impaired regional blood flow due to low cardiac output or vasomotor dysfunction. ${ }^{3,6}$

Recently, increased mean platelet volume (MPV), a routinely reported hematologic parameter of platelet size, was shown to be an indicator of platelet activation. ${ }^{7}$ Further, after coronary bypass grafting (CABG) surgery, MPV has been shown to be associated with late saphenous vein graft disease, ${ }^{8}$ postoperative atrial fibrillation, ${ }^{9}$ adverse postoperative cardiac events, and all-cause mortality. ${ }^{10}$ We previously reported an independent association between nadir postoperative platelet count and both AKI and short-term mortality after CABG surgery, suggesting platelet-related ischemic 
complications. ${ }^{5}$ Indeed, in both human and animal studies, endothelial injury reliably triggers closely related hemostatic and inflammatory mechanisms, activating platelets and ultimately resulting in microvascular sludging and consequent AKI. ${ }^{11,12}$

In the current study, we aimed to characterize the perioperative time course for changes in MPV, in particular how the changes related to platelet counts in a cohort of patients who underwent cardiac surgery. Our primary goal was to test the hypothesis that increased postoperative MPV after cardiac surgery is independently associated with postoperative AKI and mortality.

\section{Methods}

\section{Study population}

After approval (4 June 2018) by the Vanderbilt University Institutional Review Board, Vanderbilt University Medical Center, Nashville, Tennessee, we conducted this retrospective observational study on patients older than $18 \mathrm{yr}$ who underwent cardiac surgery from 12 December 2011 to 5 June 2018 . We specifically focused on the time period after 12 December 2011 when Vanderbilt University Medical Center's laboratory began measuring and reporting MPV values. From this group of 5,989 patients, we excluded 1,694 patients who had a ventricular assist device implanted or removed, surgical ablation for cardiac arrhythmias, a heart transplant, or who underwent surgery with circulatory arrest. We excluded patients with circulatory arrest given the profound impact of cardiac surgery on end-organ injury and because circulatory arrest causes hemostatic derangements that significantly increase the need for blood transfusions including platelet transfusions. The remaining 4,204 patients were included in the current study.

\section{Data collection}

The electronic medical records of all cardiac surgical patients at the Vanderbilt University Medical Center were prospectively abstracted and collected using the standardized Society of Thoracic Surgeons Adult Cardiac Surgery Database. First, we queried that database for all patients who underwent cardiac surgery at Vanderbilt University Medical Center from 12 December 2011 to 5 June 2018. Subsequently, we collected an additional set of perioperative data for these patients from two Vanderbilt University Medical Center databases: the Perioperative Data Warehouse (developed and maintained by the Vanderbilt Anesthesiology and Perioperative Informatics Research Division) and the patients' electronic medical records. Three independent investigators (A.M.R., A.A.W., and M.D.K.) verified the data quality for completeness and consistency by performing regular cross-checks between the data that was electronically captured from the Vanderbilt databases through an automated data pull from the actual electronic health records of a selected group of study patients.

\section{Clinical risk factors}

The clinical risk factors for postoperative AKI included patient characteristics, preoperative and postoperative medication use, aortic cross-clamp time, cardiopulmonary bypass duration, blood transfusions, preoperative and postoperative serum creatinine values, platelet counts, and MPV. As per institutional protocol, preoperative antiplatelet therapy with aspirin was maintained until the day before surgery, clopidogrel was discontinued between five and seven days before surgery, direct oral anticoagulants were discontinued three days before surgery, and warfarin was discontinued four days before surgery (and bridged with intravenous heparin infusion or subcutaneous dalteparin administration as appropriate). Postoperative antiplatelet therapy with aspirin, clopidogrel, and direct oral anticoagulants was typically restarted the next day after surgery, and intravenous unfractionated heparin was also restarted the next day after surgery followed by warfarin administration if necessary.

Serum creatinine was measured preoperatively and until discharge according to institutional practice; the normal range values at the Vanderbilt Clinical Pathology Laboratory were $0.4-1.0 \mathrm{mg} \cdot \mathrm{dL}^{-1}$ for women and $0.6-$ $1.3 \mathrm{mg} \cdot \mathrm{dL}^{-1}$ for men. Preoperative serum creatinine values were recorded up to 180 days before the index cardiac procedure on the day closest to, but not on, the day of surgery. Postoperative serum creatinine values were recorded daily for the first ten days postoperatively or until discharge, whichever came first. If any given day's serum creatinine values were not available, the last serum creatinine value measured on the previous day was carried over.

According to our institutional protocol, platelet counts and MPV were also measured preoperatively and for the first ten days postoperatively or until discharge, whichever came first, as described previously. ${ }^{5}$ If more than one measurement was available for platelet counts and MPV values from the same day, then the mean of these measurements was calculated and used in the subsequent analyses. The baseline MPV and platelet values were defined as the last values measured before surgery. The average postoperative MPV and platelet counts were mean values of the daily measurements before the occurrence of AKI within ten days after surgery for AKI patients, or mean values of the daily measurements during the first two 
days (i.e., the median follow-up time or time to AKI for AKI patients) for non-AKI patients. The postoperative MPV and platelet count changes were calculated as the difference between baseline values and average postoperative values.

\section{Classification of outcomes}

The primary outcome of the study was postoperative AKI, ascertained and categorized according to the Kidney Disease: Improving Global Outcomes (KDIGO) Clinical Practice Guideline for Acute Kidney Injury, with modifications because urine output was absent. ${ }^{5,13}$ Using previously developed methodology, we defined postoperative AKI using preoperative (baseline) serum creatinine values that were collected up to 180 days before surgery and all available serum creatinine values that were measured for the first 10 days after surgery or until discharge, whichever came first. We diagnosed AKI using these criteria as a $50 \%$ or greater serum creatinine increase in the first ten postoperative days or $0.3 \mathrm{mg} \cdot \mathrm{dL}^{-1}$ increase detected using a rolling 48-hr window across the ten-day postoperative period. We also staged postoperative AKI using KDIGO criteria as follows: stage I-risk, $>0.3 \mathrm{mg} \cdot \mathrm{dL}^{-1}$ or $1.5-1.99$ fold increase from baseline values; stage II-injury, 2.02.99-fold increase from baseline; stage III-failure, > 3.0fold or greater increase from baseline within ten days or $>4.0$ $\mathrm{mg} \cdot \mathrm{dL}^{-1}$ or greater increase in a rolling 48 -hr window. ${ }^{5,13}$ Patients requiring postoperative renal replacement were classified as stage III AKI.

Our secondary outcome was all-cause 30-day mortality. Survival information for the secondary outcome was ascertained from the Vanderbilt University Medical Center databases and from the Vanderbilt site-specific standardized Society of Thoracic Surgeons Adult Cardiac Surgery Database.

\section{Statistical analysis}

Patient demographics and clinical characteristics were summarized using the median [interquartile range (IQR)] for continuous variables, and percentages for categorical variables. Comparisons were made using the Wilcoxon rank sum test and the Pearson Chi square test as appropriate.

In the analysis of AKI and stage of AKI, a logistic model and a proportional odds model were used to examine the associations between changes in postoperative MPV and changes in postoperative platelet count. Postoperative AKI was a binary variable and stages of AKI were defined as an ordinal variable (no AKI, stage I, II, III), respectively, after adjusting for the clinical risk factors specified under the "clinical risk factors" section. A proportional odds model is a popular generalized linear model for modelling the dependence of an ordinal outcome variable (postoperative AKI) on its covariates (postoperative MPV and changes in postoperative platelet count; https://CRAN. R-project.org/package $=$ rms). ${ }^{14}$

In our study, we observed that patients mostly received red blood cell transfusions, and only a small number of patients received transfusion of other blood products including platelets. Therefore, in all our analyses we used "blood products administration" as a clinical variable and predictor of primary and secondary outcomes.

In the current study, any postoperative blood product administration was defined as "any blood product started after the initial surgery". Thus, we were not able to ascertain the timing of postoperative blood product administration relative to the timing of postoperative AKI. Nevertheless, to further explore the potential confounding effect of postoperative blood product administration on the association between postoperative MPV and platelet count changes, and AKI and stages of AKI, a sensitivity analysis was performed. Postoperative blood product administration was added to the AKI/AKI stage model for adjustment. Similarly, a sensitivity analysis was performed to study the potential impact of preoperative end-stage renal disease requiring renal replacement therapy on outcomes and stages of AKI. A history of preoperative renal replacement therapy was removed from the AKI/AKI stage model.

In the analysis of 30-day mortality, given the small number of events, we selected a set of risk factors according to their clinical relevance to postoperative mortality. ${ }^{5,15}$ These factors included age, sex, chronic lung disease, diabetes mellitus, cerebrovascular disease, preoperative acetylsalicylic acid use, MPV (baseline), platelet count (baseline), surgery type, intraoperative and postoperative blood product administration, postoperative average MPV and platelet count change, and postoperative AKI. Similarly, the logistic regression analysis was performed to examine the associations between postoperative MPV and platelet count change with mortality.

The nonlinear effect of postoperative platelet count and MPV changes from baseline were examined using the restricted cubic spline technique with five knots. Missing values for independent variables were imputed using multiple imputation where 10 "completed" data sets were imputed, parameters were estimated using each complete data set, and the final effect estimates and their standard errors were calculated using Rubin's rule. Adjusted odds ratios [aORs] with 95\% confidence interval $[\mathrm{CI}]$ (and corresponding $P$ value) were presented for effect size estimations. All analyses were performed using $\mathrm{R}$ version 3.6.2 ( $\mathrm{R}$ Foundation for Statistical Computing, Vienna, Austria). A two-sided significance level of 0.05 was used for statistical inference. 


\section{Results}

Demographic, clinical, and perioperative characteristics of the patients with postoperative AKI and non-AKI patients are presented in the Table. The median [IQR] age of the 4,204-patient study population was 64 [56-72] yr, and 3,008 patients $(71.5 \%)$ were male. In total, 2,676 patients (64\%) underwent isolated CABG surgery, 842 patients (20\%) underwent isolated aortic valve or mitral valve procedures, 361 patients $(9 \%)$ underwent aortic valve or mitral valve procedures with CABG surgery, and 324 patients $(8 \%)$ underwent another cardiac procedure other than CABG surgery and/or valve procedures. There were several notable differences between the groups, including age, type of procedure, history of hypertension, smoking history, beta-blocker use, and intraoperative and postoperative characteristics.

The median [IQR] postoperative MPV change was 0.21 [0.03-0.40] femtolitre (fL) for AKI patients and 0.23 [0.07-0.38] fL for non-AKI patients $(P=0.33)$, and platelet count change (reduction) was 55 [25-93] $\times$ $10^{9} \cdot \mathrm{L}^{-1}$ for AKI patients and $70[39-106] \times 10^{9} \cdot \mathrm{L}^{-1}$ for non-AKI patients $(P<0.001)$.

\section{Postoperative AKI}

As postoperative AKI was defined as ischemic kidney injury occurring within a period of ten days after surgery, four patients who died within ten days before developing AKI were excluded. One thousand three hundred and seventy-three patients $(33 \%)$ developed postoperative AKI, and $91.2 \%(n=1,252), 6 \%(n=83)$, and $2.8 \%(n=38)$ of these patients met criteria for stage I, II, and III AKI, respectively. Figure 1 shows the independent associations between clinical risk factors including patient demographics, clinical and perioperative characteristics, and postoperative average MPV and platelet count changes and postoperative AKI, based on the multivariable regression analysis. The following characteristics emerged as significant risk factors for postoperative AKI: older age, black race, higher BMI, higher baseline serum creatinine concentration, higher baseline MPV values, higher baseline platelet counts, history of diabetes mellitus, cerebrovascular disease, and intraoperative blood product administration. Although there was no evidence of association between AKI status and postoperative MPV change in unadjusted comparisons (Table), both postoperative MPV and platelet count changes had significant nonlinear associations with postoperative AKI in adjusted analyses $(P=0.007$ and $P$ $=0.003$, respectively). There was an "U" shape relationship between MPV change and the probability of having AKI. Compared with the median postoperative
MPV change (i.e., a $0.2 \mathrm{fL}$ increase), a higher increase in postoperative MPV was associated with increased risk of AKI (Fig. 2). For example, compared with patients whose average postoperative MPV value increased by $0.2 \mathrm{fL}$ (i.e., the median value) from baseline, those whose average postoperative MPV values increased by $0.8 \mathrm{fL}$ from baseline (i.e., an additional $0.6-\mathrm{fL}$ increment in average postoperative MPV) had an $80 \%$ increase in the odds of developing AKI (adjusted odds ratio [aOR], 1.80; 95\% CI,1.36 to 2.38; $P<0.001)$. Those whose average postoperative MPV value decreased by $0.4 \mathrm{fL}$ from baseline (i.e., an additional $0.6-\mathrm{fL}$ reduction in average postoperative MPV), however, had a $41 \%$ increase in odds of developing postoperative AKI (aOR, 1.41; 95\% CI,1.02 to $1.95 ; P=0.037$; Fig. 1 ).

For the AKI stage model, after adjusting for other demographic and clinical characteristics, patients with a postoperative MPV change greater than $0.2 \mathrm{fL}$ from baseline remained at elevated odds of progressing to a higher severity of postoperative AKI (aOR, 1.66; 95\% CI, 1.28 to $2.16 ; P<0.001$; Fig. 3 ).

In separate analyses, we explored the potential effect of postoperative blood product administration on the associations between postoperative MPV and platelet counts changes and incidence and severity of AKI. After adjusting for postoperative blood product administration, the association between postoperative MPV and platelet count changes and AKI and stages of AKI remained unchanged (available in the Electronic Supplementary Material [ESM] as eFigs 1 and 2). Further, we found that patients receiving intraoperative transfusions $v s$ no transfusion of blood products experienced a greater median [IQR] reduction of postoperative platelets count (83 [44-125] vs 57 [31-88], respectively; $P<0.001$ ). Similarly, patients who received postoperative blood transfusions had a greater postoperative platelet count reduction compared with those who did not receive postoperative blood transfusions (78 [45-121] vs 63 [3396], respectively; $P<0.001)$.

In a subsequent sensitivity analysis, we excluded patients with a history of preoperative renal replacement therapy to explore its potential impact on the association between postoperative MPV and platelet count changes and AKI and stages of AKI. Our results remained consistent with similar effect estimates (available in the ESM as eFigs 1 and 2).

Postoperative mortality

Ninety-two of the 4,133 patients with available data $(2.02 \%)$ died within 30 days of surgery (ESM eTable 1), and the causes of death are listed in ESM eTable 2. In adjusted analysis, older age, higher MPV at baseline, 


\section{Acute Kidney Injury}

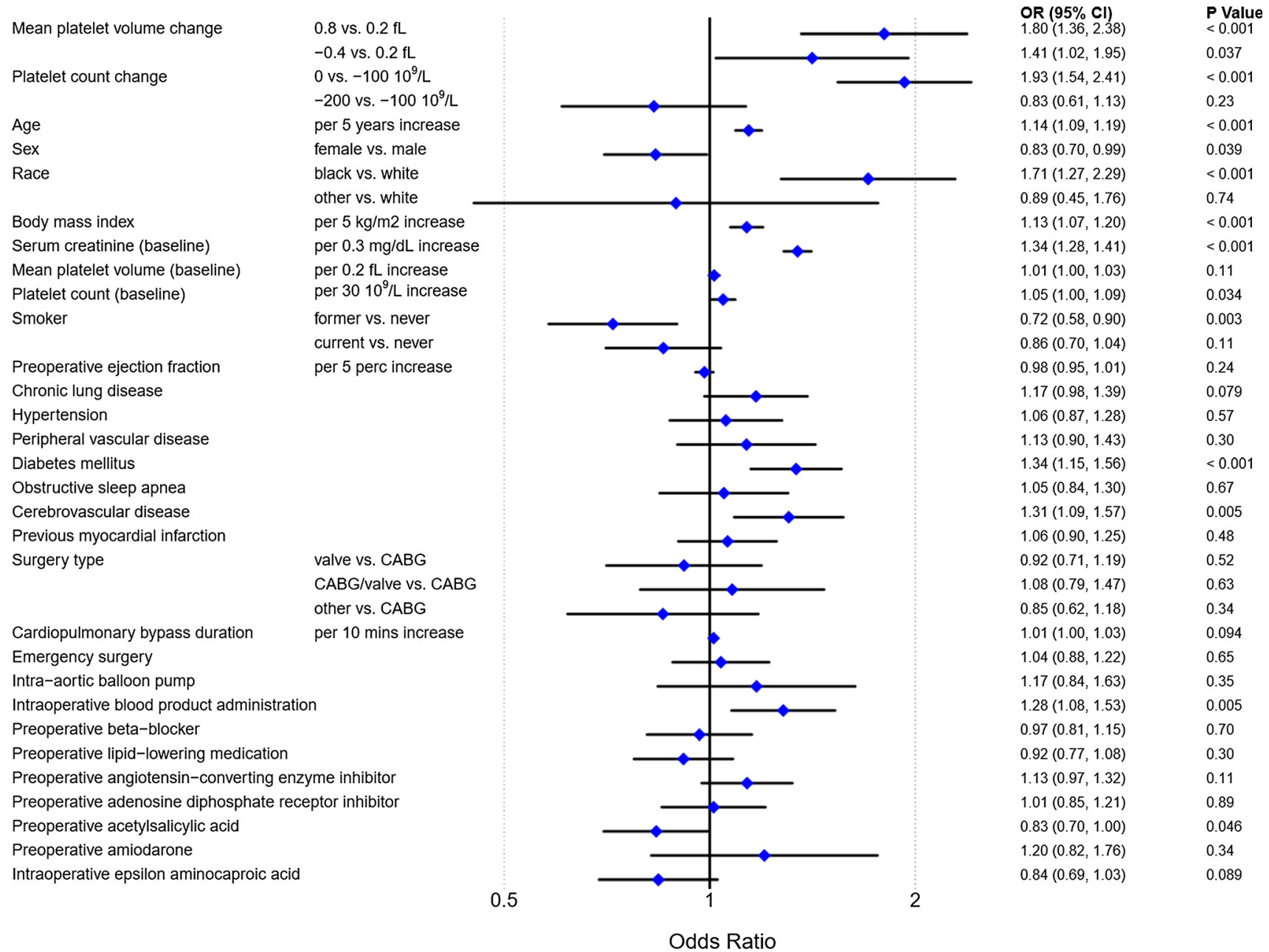

Fig. 1 The estimated odds ratio of having acute kidney injury for each risk factor. The diamond shape and the horizontal segment represent the odds ratio and 95\% confidence interval, respectively. All

intraoperative and postoperative blood product administration, and postoperative AKI were significantly associated with elevated 30-day all-cause mortality (Fig. 4). Higher MPV at baseline was not significantly associated with a higher risk for 30-day all-cause mortality. In contrast, lower platelet count at baseline was associated with higher probability of 30-day all-cause mortality. The postoperative MPV change was not associated with 30-day all-cause mortality (Fig. 4). There was a significant nonlinear relationship between postoperative platelet count change and 30-day mortality (Figs 2 and 4). Compared with patients whose postoperative platelet count were unchanged, however, those who had a $100 \times$ $10^{9} \cdot \mathrm{L}^{-1}$ decrease had a higher odds of mortality (OR, 3.96; 95\% CI, 2.26 to 6.96; $P<0.001$; Fig. 4). effects were estimated from the multivariable logistic model. CABG = coronary artery bypass grafting surgery.

\section{Discussion}

In this single-centre retrospective observational study, we found a significant association between postoperative increase in MPV and postoperative AKI. Importantly, the magnitude of change in MPV correlated significantly with the severity of kidney injury. Postoperative increase in MPV, however, was not significantly associated with allcause 30-day mortality.

Cardiac surgery is a major cause of acquired thrombocytopenia, predominantly due to excessive platelet consumption. ${ }^{16}$ The subsequent reactive thrombopoietic response in the postoperative period releases large numbers of immature platelets into the circulation. Because of an increase in RNA content in these platelets, they have a reticulated appearance under light microscopy, so are referred to as reticulated platelets. ${ }^{17}$ 

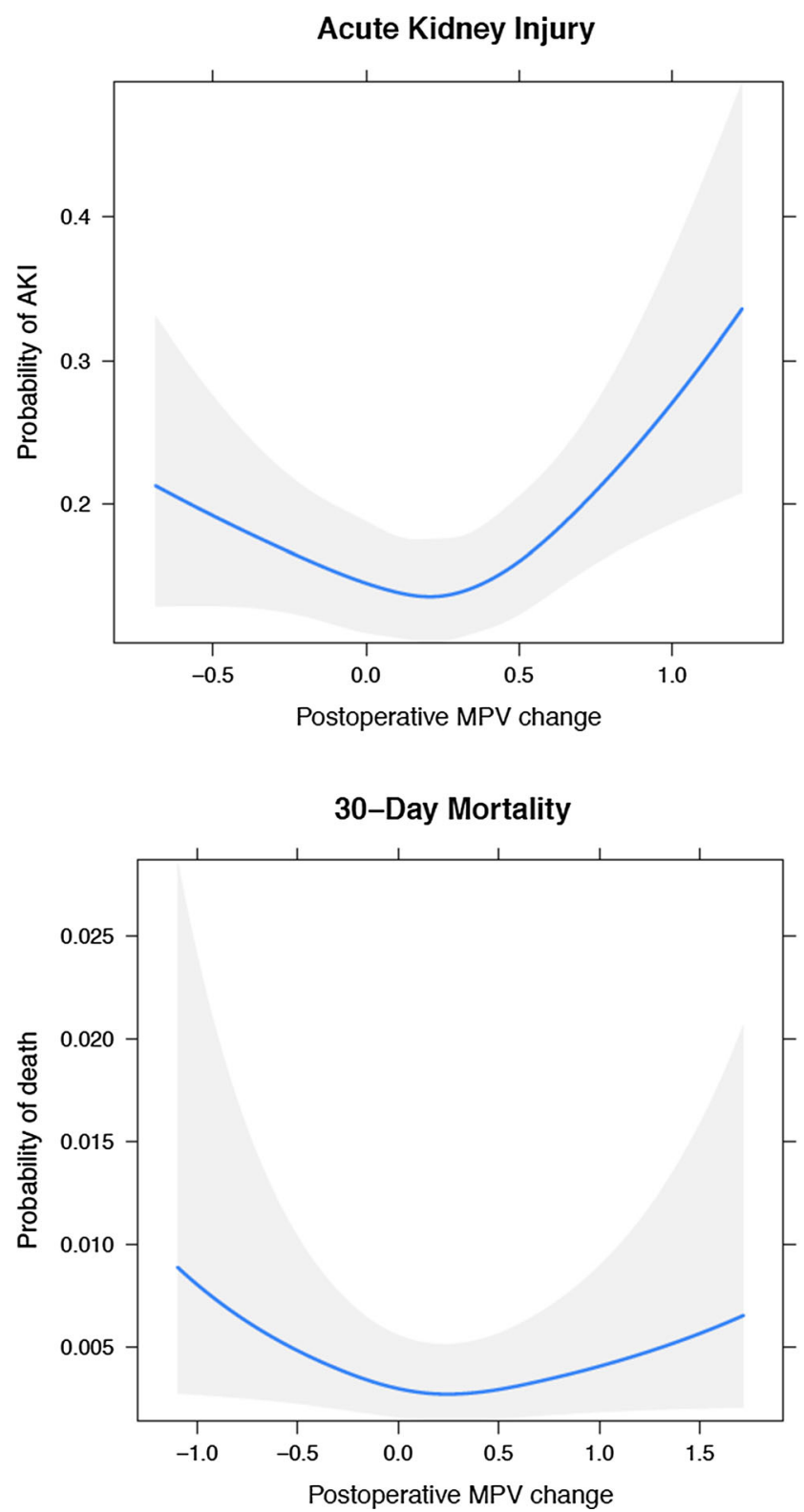

Fig. 2 Probability of having acute kidney injury (upper panels) and probability of 30-day all cause death (lower panels) conditional on postoperative mean platelet volume change and platelet count change. The blue line is probabilities calculated from the logistic models. The

Reticulated platelets are larger, have a higher density of alpha granules, and exhibit significantly greater reactivity than mature platelets. The MPV, an automated measure of platelet size, has increasingly been associated with adverse outcomes such as acute coronary syndrome, antiplatelet drug resistance, and even inflammation. ${ }^{15}$ The results of our study show that a postoperative increase in MPV after cardiac surgery was associated with both a higher risk and severity of AKI, but there was no association between postoperative increase in MPV and mortality. This observation may indicate that once AKI develops
Acute Kidney Injury

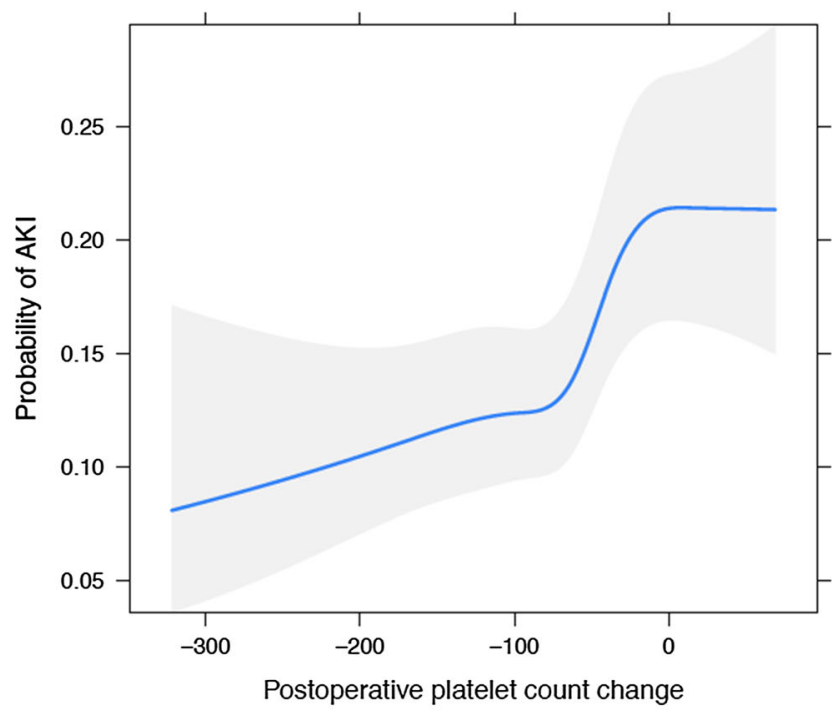

30-Day Mortality

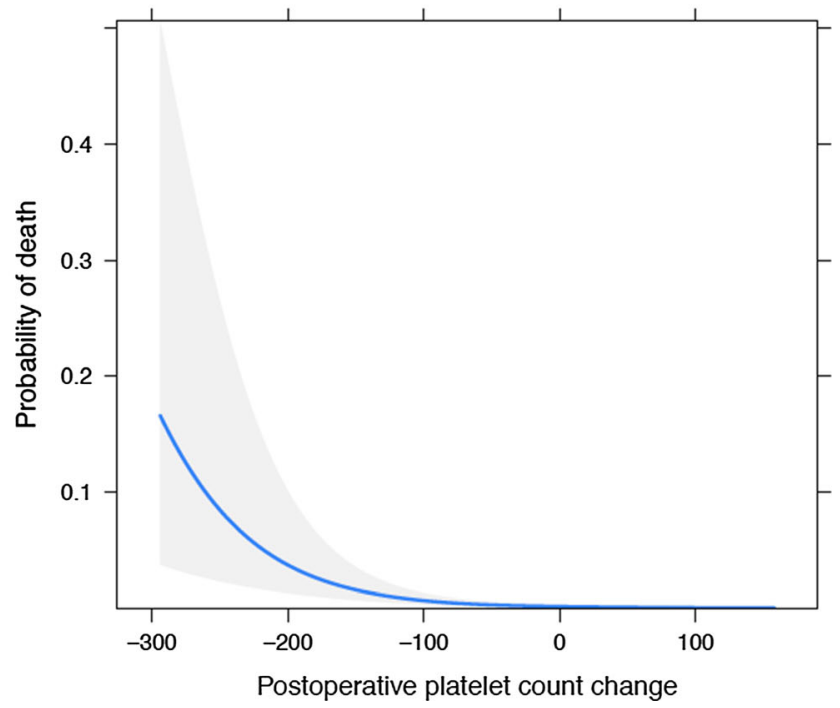

shaded area in gray is the $95 \%$ confidence band. Covariates not plotted were set to their median or mode values. AKI = acute kidney injury; $\mathrm{BL}=$ baseline; $\mathrm{MPV}=$ mean platelet volume; $30-\mathrm{D}=30$-day;

secondary to platelet activation after cardiac surgery, it emerges as a stronger predictor of postoperative mortality than the initial platelet activation. Alternatively, MPV may not have emerged as a predictor of mortality because of low statistical power in our smaller cohort of patients who suffered more severe stages of AKI. For example, while 1,373 patients $(33 \%)$ developed AKI, 91.2\% $(n=1,252)$ suffered stage I AKI whereas only $6 \%(n=83)$ and $2.8 \%(n$ $=38$ ) developed stages II and III, respectively. Also, in the current study, our cohort was comprised of 2,676 patients (64\%) who underwent isolated CABG surgery, 842 patients 


\section{Acute Kidney Injury Stage}

\begin{tabular}{|c|c|c|c|c|}
\hline Mean platelet volume change & 0.8 vs. $0.2 \mathrm{fL}$ & & $\begin{array}{l}\text { OR }(95 \% \text { Cl) } \\
1.66(1.28,2.16)\end{array}$ & $\begin{array}{l}\text { P Value } \\
<0.001\end{array}$ \\
\hline & -0.4 vs. $0.2 \mathrm{fL}$ & & $1.31(0.97,1.78)$ & 0.076 \\
\hline \multirow[t]{2}{*}{ Platelet count change } & 0 vs. $-10010^{9} / \mathrm{L}$ & & $1.85(1.49,2.29)$ & $<0.001$ \\
\hline & -200 vs. $-10010^{9} / L$ & & $0.88(0.66,1.18)$ & 0.41 \\
\hline Age & per 5 years increase & $\sim$ & $1.14(1.09,1.19)$ & $<0.001$ \\
\hline Sex & female vs. male & & $0.75(0.63,0.88)$ & $<0.001$ \\
\hline \multirow[t]{2}{*}{ Race } & black vs. white & & $1.79(1.36,2.35)$ & $<0.001$ \\
\hline & other vs. white & & $0.90(0.49,1.69)$ & 0.75 \\
\hline Body mass index & per $5 \mathrm{~kg} / \mathrm{m} 2$ increase & 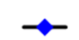 & $1.14(1.08,1.19)$ & $<0.001$ \\
\hline Serum creatinine (baseline) & per $0.3 \mathrm{mg} / \mathrm{dL}$ increase & • & $1.11(1.09,1.13)$ & $<0.001$ \\
\hline Mean platelet volume (baseline) & per $0.2 \mathrm{fL}$ increase & & $1.02(1.00,1.04)$ & 0.038 \\
\hline Platelet count (baseline) & per $3010^{9} / \mathrm{L}$ increase & & $1.04(1.00,1.08)$ & 0.043 \\
\hline \multirow[t]{2}{*}{ Smoker } & former vs. never & & $0.73(0.59,0.90)$ & 0.003 \\
\hline & current vs. never & & $0.86(0.71,1.04)$ & 0.11 \\
\hline Preoperative ejection fraction & per 5 perc increase & & $0.98(0.95,1.01)$ & 0.17 \\
\hline \multicolumn{2}{|l|}{ Chronic lung disease } & & $1.16(0.98,1.37)$ & 0.082 \\
\hline \multicolumn{2}{|l|}{ Hypertension } & & $1.12(0.93,1.34)$ & 0.24 \\
\hline \multicolumn{2}{|l|}{ Peripheral vascular disease } & & $1.17(0.94,1.45)$ & 0.17 \\
\hline \multicolumn{2}{|l|}{ Diabetes mellitus } & & $1.43(1.24,1.65)$ & $<0.001$ \\
\hline \multicolumn{2}{|l|}{ Obstructive sleep apnea } & & $1.07(0.87,1.32)$ & 0.51 \\
\hline \multicolumn{2}{|l|}{ Cerebrovascular disease } & & $1.38(1.16,1.64)$ & $<0.001$ \\
\hline \multicolumn{2}{|l|}{ Previous myocardial infarction } & & $1.07(0.91,1.26)$ & 0.39 \\
\hline \multirow[t]{3}{*}{ Surgery type } & valve vs. CABG & & $1.01(0.78,1.29)$ & 0.97 \\
\hline & CABG/valve vs. CABG & & $1.07(0.80,1.44)$ & 0.64 \\
\hline & other vs. CABG & & $1.02(0.76,1.38)$ & 0.88 \\
\hline \multirow{2}{*}{\multicolumn{2}{|c|}{$\begin{array}{l}\text { Cardiopulmonary bypass duration } \\
\text { Emergency surgery }\end{array}$}} & & $1.01(1.00,1.03)$ & 0.14 \\
\hline & & & $1.06(0.91,1.24)$ & 0.46 \\
\hline \multicolumn{2}{|c|}{ Intra-aortic balloon pump } & & $1.26(0.91,1.73)$ & 0.16 \\
\hline \multicolumn{2}{|c|}{ Intraoperative blood product administration } & & $1.39(1.18,1.65)$ & $<0.001$ \\
\hline \multicolumn{2}{|c|}{ Preoperative beta-blocker } & & $0.98(0.83,1.17)$ & 0.85 \\
\hline \multicolumn{2}{|l|}{ Preoperative lipid-lowering medication } & 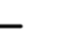 & $0.91(0.78,1.07)$ & 0.27 \\
\hline \multicolumn{2}{|c|}{ Preoperative angiotensin-converting enzyme inhibitor } & & $1.06(0.91,1.22)$ & 0.46 \\
\hline \multicolumn{2}{|c|}{ Preoperative adenosine diphosphate receptor inhibitor } & & $1.03(0.87,1.21)$ & 0.75 \\
\hline \multicolumn{2}{|c|}{ Preoperative acetylsalicylic acid } & & $0.85(0.72,1.01)$ & 0.057 \\
\hline \multicolumn{2}{|l|}{ Preoperative amiodarone } & & $1.22(0.85,1.75)$ & 0.28 \\
\hline \multicolumn{2}{|c|}{ Intraoperative epsilon aminocaproic acid } & & $0.84(0.69,1.02)$ & 0.074 \\
\hline & 0.5 & & & \\
\hline
\end{tabular}

Fig. 3 The estimated odds ratio of severity (stages) of acute kidney injury for each risk factor. The diamond shape and the horizontal segment represent the odds ratio and 95\% confidence interval, respectively. The odds ratios are for increase or decrease in risk for

(20\%) who underwent isolated aortic valve- or mitral valve procedures, 361 patients $(9 \%)$ who underwent aortic valve or mitral valve procedures with CABG surgery, and 324 patients $(8 \%)$ who underwent another cardiac procedure other than CABG surgery and/or valve procedures. Mortality rates and predictors of mortality vary depending on the specific cardiac procedure; therefore, the effects of procedure on the relationship between MPV and mortality should be investigated, also in larger study populations with sufficient statistical power to address mortality.

The MPV values tend to be higher in conditions with acute increased platelet use and turnover, such as myocardial infarctions, unstable angina, stroke, and surgical procedures. ${ }^{18}$ Further, there is increased production of hemostatic factors and granules, higher worsening stages of acute kidney injury. All effects were estimated from the proportional odds model. $\mathrm{CABG}=$ coronary artery bypass grafting surgery.

expression of receptors including cyclooxygenase-1, and faster responses to collagen exposure. On the other hand, in diseases with chronic inflammation, such as asthma, chronic obstructive pulmonary disease, rheumatoid arthritis, and ulcerative colitis, MPV values are low. This is likely due to the effect of long-term cytokine exposure, including interleukin-6, on thrombopoeisis. ${ }^{19,20}$ In AKI specifically, platelet activation likely plays a key role in its early pathogenesis. As such, previous studies have shown an association between higher MPV values and AKI. ${ }^{21}$

The results of our study are consistent with those of Aydinli et al. who found that after CABG surgery, valvular surgery, or combined CABG and valve surgery, increased MPV predicted combined adverse events, including renal complications and 30-day mortality. ${ }^{22}$ Interestingly, they found that using MPV in conjunction with neutrophil-to- 


\section{0-Day Mortality}

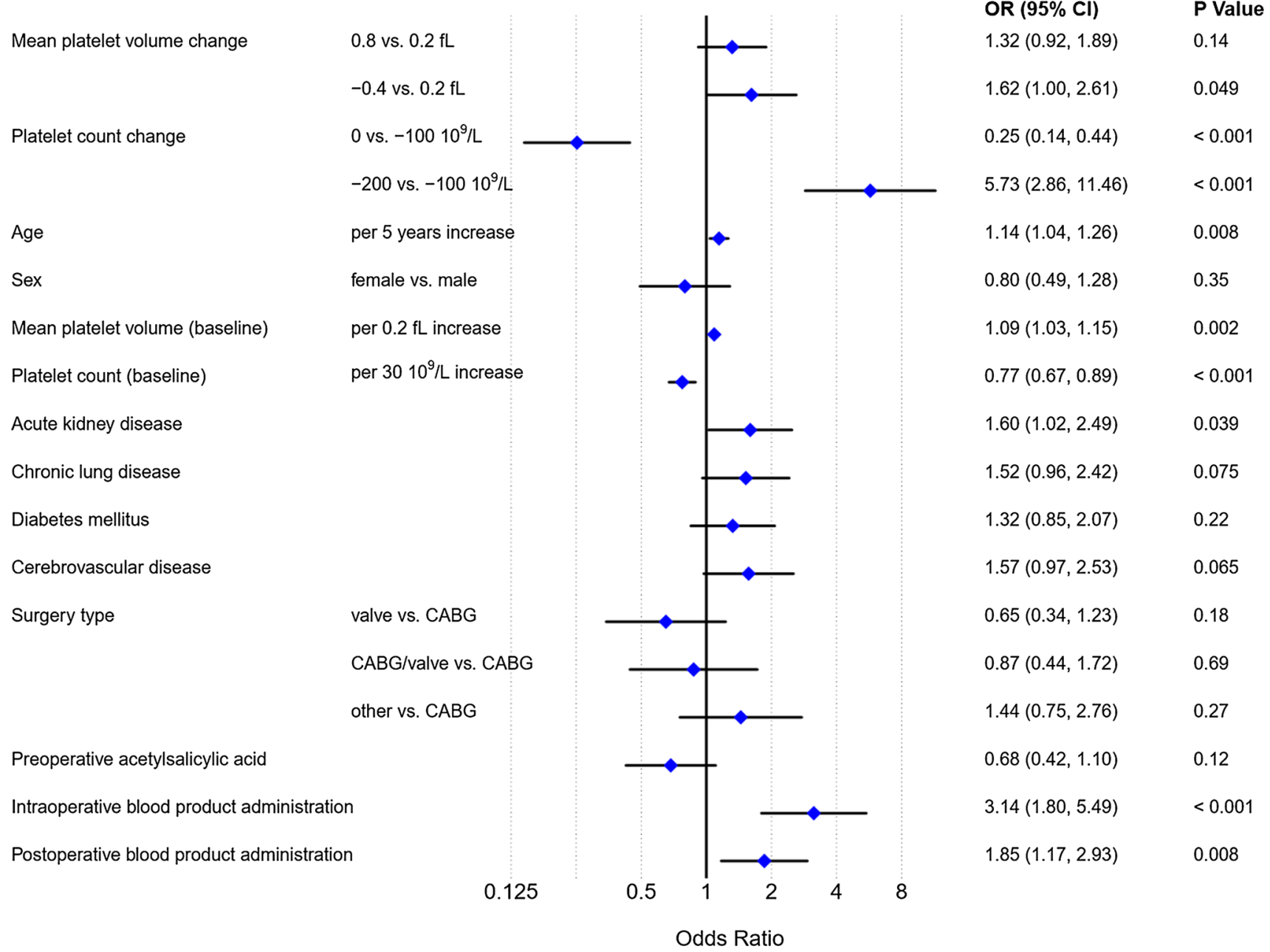

Fig. 4 The estimated odds ratio of 30-day all-cause death for each risk factor. The diamond shape and the horizontal segment represent the odds ratio and $95 \%$ confidence interval, respectively. All effects

lymphocyte ratio and red cell distribution width increased the accuracy of adverse outcomes prediction. Indeed, these observations support the mechanism for surgery-associated activation of the innate immune system, in which platelet activation plays a significant role in the early initiation of the inflammatory response reaction. The subsequent aggregation of platelets and release of platelet factors, including pro-IL-1 $\beta$, platelet-derived growth factor, and serotonin, facilitate leukocyte and neutrophil recruitment. This results in extensive inflammation, including neutrophil-mediated cytotoxicity, oxidant production, and arachidonic metabolism, which is linked to thrombosis. ${ }^{23}$

We showed a nonlinear relationship between platelet count and MPV values. This association has been previously described, ${ }^{24}$ and it has been hypothesized that the nonlinearity could be due to interactions between platelet size and thrombocytopenia. ${ }^{25}$ Studies have shown were estimated from the multivariable logistic model. $\mathrm{CABG}=$ coronary artery bypass grafting surgery.

that the degree and mechanism (i.e., hypoproduction or hyperdestruction) of thrombocytopenia directly alter MPV. ${ }^{21}$ Patients with hyperdestruction of platelets, for example, tend to have higher MPV values and lower platelet counts. In contrast, patients with inflammatory states tend to have higher MPV values and higher platelet counts. ${ }^{26}$ Indeed, in support of the latter observation, our findings showed that patients who had higher average baseline and postoperative MPV values on average had a much lower platelet count reduction (less than $100 \times$ $10^{9} \cdot \mathrm{L}^{-1}$ ) after surgery, and this was associated with a higher odds for postoperative AKI.

\section{Risk factors for AKI}

We confirmed the prognostic value of many previously identified risk factors for predicting postoperative AKI 
Table Demographic and clinical characteristics of the study population, $n=4,204$

\begin{tabular}{|c|c|c|c|c|}
\hline Predictors & $\begin{array}{l}\text { Number of } \\
\text { available }\end{array}$ & $\begin{array}{l}\text { Patients without AKI } \\
(n=2,827)\end{array}$ & $\begin{array}{l}\text { Patients with AKI } \\
(n=1,373)\end{array}$ & $P$ value \\
\hline \multicolumn{5}{|l|}{ Demographics } \\
\hline Age & 4199 & $64[56-71]$ & $66[58-73]$ & $<0.001^{1}$ \\
\hline Sex & 4199 & & & $0.019^{2}$ \\
\hline Female & & $836(30)$ & $358(26)$ & \\
\hline Male & & $1991(70)$ & $1014(74)$ & \\
\hline Race & 3974 & & & $<0.001^{2}$ \\
\hline White & & $2512(94)$ & $1136(88)$ & \\
\hline African American & & $129(5)$ & $141(11)$ & \\
\hline Other & & $41(2)$ & $15(1)$ & \\
\hline Body mass index, $\mathrm{kg} \cdot \mathrm{m}^{-2}$ & 4196 & $28.8[25.3-33.1]$ & $29.9[25.7-34.5]$ & $<0.001^{1}$ \\
\hline \multicolumn{5}{|l|}{ Medical history } \\
\hline Chronic lung disease & 4199 & $592(21)$ & $354(26)$ & $<0.001^{2}$ \\
\hline Hypertension & 4199 & $2152(76)$ & $1133(83)$ & $<0.001^{2}$ \\
\hline Prior heart failure & 4199 & $376(13)$ & $244(18)$ & $<0.001^{2}$ \\
\hline Peripheral vascular disease & 4199 & $256(9)$ & $181(13)$ & $<0.001^{2}$ \\
\hline Diabetes mellitus & 4199 & $1097(39)$ & $729(53)$ & $<0.001^{2}$ \\
\hline Obstructive sleep apnea & 4199 & $347(12)$ & $201(15)$ & $0.032^{2}$ \\
\hline Cardiovascular disease & 4199 & 444 (16) & $302(22)$ & $<0.001^{2}$ \\
\hline Smoker & 4198 & & & $0.001^{2}$ \\
\hline Never & & $1676(59)$ & $894(65)$ & \\
\hline Former & & $450(16)$ & $194(14)$ & \\
\hline Current & & $700(25)$ & $284(21)$ & \\
\hline Previous myocardial infarction & 4199 & $1033(37)$ & $565(41)$ & $0.004^{2}$ \\
\hline \multicolumn{5}{|l|}{ Preoperative medication } \\
\hline Acetylsalicylic acid & 4199 & $2090(74)$ & $994(72)$ & $0.31^{2}$ \\
\hline $\begin{array}{l}\text { Adenosine diphosphate inhibitors, } 5 \text { days before } \\
\text { surgery }\end{array}$ & 4199 & $673(24)$ & $370(27)$ & $0.026^{2}$ \\
\hline Amiodarone & 2072 & $82(6)$ & $54(9)$ & $0.006^{2}$ \\
\hline Angiotensin-converting enzyme inhibitors & 4199 & $1155(41)$ & $573(42)$ & $0.57^{2}$ \\
\hline Anticoagulants & 4199 & $933(33)$ & $478(35)$ & $0.24^{2}$ \\
\hline Anti-platelets, 5 days before surgery & 4198 & $70(2)$ & $28(2)$ & $0.38^{2}$ \\
\hline Beta blockers & 4199 & $2026(72)$ & $1020(74)$ & $0.068^{2}$ \\
\hline Coumadin & 4199 & $16(1)$ & $7(1)$ & $0.82^{2}$ \\
\hline Glycoprotein IIb/IIIa inhibitor & 4199 & $17(1)$ & $0(0)$ & $0.004^{2}$ \\
\hline Lipid-lowering medications & 4199 & $1878(66)$ & $914(67)$ & $0.9^{2}$ \\
\hline \multicolumn{5}{|l|}{ Intraoperative characteristics and medications } \\
\hline Timing of surgery & 4199 & & & $0.06^{2}$ \\
\hline Elective & & $1744(62)$ & 805 (59) & \\
\hline Emergent & & $1083(38)$ & $567(41)$ & \\
\hline Type of surgery & 4200 & & & $0.086^{2}$ \\
\hline Isolated CABG & & $1805(64)$ & $870(63)$ & \\
\hline Valve only & & $585(21)$ & $254(19)$ & \\
\hline CABG and valve & & $226(8)$ & $113(8)$ & \\
\hline Other & & $211(7)$ & $113(8)$ & \\
\hline Operative approach & 4189 & & & $0.91^{2}$ \\
\hline Full conventional sternotomy & & $2539(90)$ & $1231(90)$ & \\
\hline Other & & $281(10)$ & $138(10)$ & \\
\hline Duration of cardiopulmonary bypass total, mins & & $71[0-115]$ & $66[0-121]$ & $0.92^{1}$ \\
\hline
\end{tabular}


Table continued

\begin{tabular}{|c|c|c|c|c|}
\hline Predictors & $\begin{array}{l}\text { Number of } \\
\text { available }\end{array}$ & $\begin{array}{l}\text { Patients without AKI } \\
(n=2,827)\end{array}$ & $\begin{array}{l}\text { Patients with AKI } \\
(n=1,373)\end{array}$ & $P$ value \\
\hline Cross-clamp duration, mins & & 74 [56-99] & $77[56-110]$ & $0.04^{1}$ \\
\hline Intra-aortic balloon pump use & 4199 & $133(5)$ & $86(6)$ & $0.033^{2}$ \\
\hline Epsilon aminocaproic acid use & 4198 & $1509(53)$ & $713(52)$ & $0.38^{2}$ \\
\hline Tranexamic acid use & 4197 & $21(1)$ & $13(1)$ & $0.49^{2}$ \\
\hline Intraoperative blood products administration & & $1011(36)$ & $636(46)$ & $<0.001^{2}$ \\
\hline \multicolumn{5}{|c|}{ Preoperative hemodynamic and laboratory tests } \\
\hline Preoperative ejection fraction, $\%$ & 4193 & $55[50-60)$ & $55[45-60]$ & $<0.001^{1}$ \\
\hline Mean platelet volume, fL & 3856 & $10.25[9.70-10.85)$ & $10.35[9.80-11.00]$ & $<0.001^{1}$ \\
\hline Platelet count, $10^{9} \mathrm{~L}^{-1}$ & 3540 & $220(181-266)$ & 209 [169-257] & $<0.001^{1}$ \\
\hline Creatinine, $\mathrm{mg} \cdot \mathrm{dL}^{-1}$ & 4196 & $0.96[0.83-1.14)$ & $1.23[0.99-1.68]$ & $<0.001^{1}$ \\
\hline \multicolumn{5}{|l|}{ Postoperative laboratory tests } \\
\hline Average mean platelet volume, fL & & $10.48[9.95-11.08)$ & $10.60[10.07-11.19]$ & $<0.001^{1}$ \\
\hline Average mean platelet volume change & & $0.23[0.07-0.38]$ & $0.21[0.03-0.40]$ & $0.33^{1}$ \\
\hline Average platelet count, $10^{9} \mathrm{~L}^{-1}$ & & 146 [111-182] & $148[115-186]$ & $0.1^{1}$ \\
\hline Average platelet count change & & $-70[-106$ to -39$]$ & $-55[-93$ to -25$]$ & $<0.001^{1}$ \\
\hline Peak creatinine, $\mathrm{mg} \cdot \mathrm{dL}^{-1}$ & 4158 & $0.98[0.84-1.15]$ & $1.70[1.34-2.41]$ & $<0.001^{1}$ \\
\hline
\end{tabular}

Postoperative blood products administration

All data are represented either as median [interquartile range] or number $(\%)$. Data was analyzed with ${ }^{1}$ Wilcoxon rank sum test or ${ }^{2}$ Pearson test with $P<0.05$ as significant. AKI = acute kidney injury; CABG = coronary artery bypass grafting surgery; $\mathrm{fL}=$ femtolitre.

after cardiac surgery, ${ }^{5}$ including advanced age, black race, higher body mass index, higher baseline platelet counts, cardiovascular disease, and administration of intraoperative and postoperative blood products. Importantly, we observed that most of these risk factors were associated with magnitude of postoperative MPV and platelet count changes.

Advanced age, higher body mass index, and cardiovascular disease have previously been found to increase the risk for postoperative AKI. ${ }^{5,27}$ Patients with these risk factors often have widespread systemic vascular disease resulting in impaired renal blood flow and increased susceptibility to AKI in the perioperative period. $^{28}$ Given that platelets play a crucial role in the pathogenesis of most vascular disease conditions and their subsequent end-organ ischemic complications, it would be reasonable to investigate roles of antiplatelet therapy in patients at high-risk for AKI.

Our finding that higher baseline platelet counts were associated with a higher risk for postoperative AKI highlights the role of preexisting systemic inflammation and platelet activation and their potential impact on postcardiac surgery-related acute inflammatory response reaction and higher risk for complications including AKI. ${ }^{29}$ Indeed, these findings provide further support to the concept of using increased baseline platelet counts along with other inflammatory biomarkers to identify highrisk cardiac surgery patients prior to surgery who could benefit from risk management strategies aimed at mitigating the systemic inflammatory response and platelet activation. ${ }^{30}$ Of note, increased baseline MPV values indicated an elevated risk for postoperative AKI, but this association did not remain statistically significant after we adjusted for relevant clinical variables. Thus, future research is needed to investigate this complex association between preoperative MPV and platelet counts, clinical risk factors, and postoperative AKI.

Previous reports by us ${ }^{5}$ and others ${ }^{31}$ confirmed the link between intraoperative transfusion of packed red blood cells and postoperative AKI. In our current study, however, we cannot provide evidence of the causative effect of blood transfusion; the increased risk for AKI may originate from the effects of preceding anemia, preexisting kidney injury, intraoperative hypotension and volume shifts, medication use, or pathophysiologic changes triggered by the transfusion itself. ${ }^{32}$

\section{Risk factors for mortality}

Following CABG surgery, MPV has been associated with late saphenous vein graft disease, ${ }^{8}$ postoperative adverse cardiac events and all-cause mortality, ${ }^{10}$ and postoperative atrial fibrillation. ${ }^{9}$ Whereas we found a relationship between postoperative thrombocytopenia and mortality, changes in MPV after surgery were not a significant predictor of death. This observation may reflect the 
heterogeneous cohort of patients undergoing cardiac surgery with nearly one third having non-coronary cardiac procedures. While the pathophysiologic underpinning of coronary disease and atherosclerosis involves triggers and mediators of a systemic inflammatory responses leading to increased platelet reactivity and involving increased MPV (an indicator of platelet reactivity), the pathogenesis of valvular heart disease is less related to platelets. ${ }^{5,21,33,34}$ Furthermore, as shown in the Table, both bleeding and transfusion were significantly more common in non-CABG and in more complex procedures. Transfusion of blood products (namely platelets) and hemodilution could affect the postoperative MPV.

\section{Limitations}

Given its retrospective design, our study has several limitations. Information on some important clinical risk factors of postoperative AKI and mortality were not prospectively collected. Instead, we relied on electronic medical records and physician documentation as sources of additional information on clinical risk factors, medication use, blood transfusion, and laboratory data. Therefore, the potential effects and the magnitude of effects of certain clinical risk factors, medication use, blood transfusion, and laboratory data may have been under- or overestimated. Nevertheless, their observed predictive effects were similar to those reported from previous studies. ${ }^{5,35}$

Because this study was conducted at a single tertiary academic centre, its findings may not be fully applicable to similar institutions with different mixes of cardiac surgical populations, perioperative management practices, and levels of expertise.

The reference range for normal MPV values used in our study was based on the definition set by the Vanderbilt Clinical Pathology Laboratory. This definition for a normal reference range is slightly different from other studies, and it was not specifically reported for male and female patients separately. ${ }^{36}$ Furthermore, there is evidence that normal reference intervals recommended by different manufacturers of hematological analyzers could be different, and thus, laboratories often develop their own normal reference ranges. ${ }^{37,38}$ Therefore, we opted to use an unbiased approach of measuring and studying the effect average change of postoperative MPV values compared with baseline with AKI and mortality.

No specific protocols were used to guide intra- and postoperative transfusions or anticoagulation therapies perioperatively. Of note, there is a paucity of data on how intra- and postoperative transfusions-in particular, platelet transfusions-could influence the postoperative course of MPV and the corresponding platelet counts after cardiac surgery. Also, as in our previous study, testing in our centre for heparin-induced thrombocytopenia was left to the discretion of the intensive care unit team. ${ }^{5}$ Moreover, given the retrospective design of our study, we were not able to obtain consistent data about the presence of heparin-induced thrombocytopenia or the results of laboratory screening tests for human platelet factor 4/ heparin complex antibodies. According to our previous study, the incidence of heparin-induced thrombocytopenia was low $(0.3 \%)$, and excluding these patients from association analyses did not change the association between postoperative thrombocytopenia and AKI. ${ }^{5}$

Finally, assumptions about the etiology of elevated MPV values and postoperative thrombocytopenia cannot be made. Changes in MPV values and in platelet counts have been attributed to several different acute and chronic conditions or medication use. Indeed, when it comes to the classification of platelet disorders, patients can have high, low, or normal MPV values, and high, low, or normal platelet counts. ${ }^{39,40}$ In the perioperative setting, increased destruction, hemodilution, and sequestration, and decreased production can all influence MPV values and platelet counts. ${ }^{5,41}$ As such, changes may not necessarily result in concurrent increases in MPV value and decreases in platelet counts. ${ }^{39,40}$ Although we previously showed a significant association between leukocyte and platelet activation and ischemic complications after cardiac surgery as a potential indication for platelet activation, ${ }^{42}$ we did not measure markers of inflammation and microthrombosis in our current study. Future studies will have to define and refine the context of platelet activation, changes in MPV, platelet counts, and inflammation-related ischemic complications after cardiac surgery.

In conclusion, our findings suggest an independent association between the magnitude of postoperative MPV changes and the development of postoperative AKI and severity of AKI. This complex association requires further investigation to characterize specific mechanisms that lead to postoperative AKI and mortality, and how routinely measured MPV and changes in MPV could be integrated in the perioperative patient management and decision-making about targeted therapies to reduce platelet-related ischemic complications in the setting of cardiac surgery.

Author contributions Abinaya Ramaksihnan, Cynthia Zheng, and Miklos D. Kertai contributed to all aspects of this manuscript, including study conception and design; acquisition, analysis, and interpretation of data; and drafting the article. Manuel L Fontes, Frederic W. Lombard, Frederic T Billings 4th, Mia Pretorius, Tarek S. Absi, and Ashish S. Shah contributed to the study conception and design, and interpretation of data and drafting the manuscript. Austin A. Woolard, Jonathan P. Wanderer, and Rushikesh Vyas contributed to the acquisition of data. Yaping Shi and Mathew S. Shotwell contributed to the analysis and interpretation of data, and drafting the article. 
Disclosures None.

Funding statement None.

Editorial responsibility This submission was handled by Dr. Hilary P. Grocott, Editor-in-Chief, Canadian Journal of Anesthesia.

\section{References}

1. D'Agostino RS, Jacobs JP, Badhwar V, et al. The Society of Thoracic Surgeons Adult Cardiac Surgery Database: 2019 update on outcomes and quality. Ann Thorac Surg 2019; 107: 24-32.

2. O'Neal JB, Shaw AD, Billings FT 4th. Acute kidney injury following cardiac surgery: current understanding and future directions. Crit Care 2016; DOI: https://doi.org/10.1186/s13054016-1352-z.

3. Kindzelski BA, Corcoran P, Siegenthaler MP, Horvath KA. Postoperative acute kidney injury following intraoperative blood product transfusions during cardiac surgery. Perfusion 2018; 33: 62-70.

4. Parolari A, Pesce LL, Pacini D, et al. Risk factors for perioperative acute kidney injury after adult cardiac surgery: role of perioperative management. Ann Thorac Surg 2012; 93: 584-91.

5. Kertai MD, Zhou S, Karhausen JA, et al. Platelet counts, acute kidney injury, and mortality after coronary artery bypass grafting surgery. Anesthesiology 2016; 124: 339-52.

6. Wang $Y$, Bellomo $R$. Cardiac surgery-associated acute kidney injury: risk factors, pathophysiology and treatment. Nat Rev Nephrol 2017; 13: 697-711.

7. Gasparyan AY, Ayvazyan L, Mikhailidis DP, Kitas GD. Mean platelet volume: a link between thrombosis and inflammation? Curr Pharm Des 2011; 17: 47-58.

8. Tavil Y, Sen N, Yazici HU, et al. Relationship between elevated platelet volume and saphenous vein graft disease. Clin Invest Med 2010; 33: E161-7.

9. Erdem K, Ayhan $S$, Ozturk $S$, et al. Usefulness of the mean platelet volume for predicting new-onset atrial fibrillation after isolated coronary artery bypass grafting. Platelets 2014; 25: 23-6.

10. Unal EU, Ozen A, Kocabeyoglu S, et al. Mean platelet volume may predict early clinical outcome after coronary artery bypass grafting. J Cardiothorac Surg 2013; DOI: https://doi.org/10.1186/ 1749-8090-8-91.

11. Thachil J, Warkentin TE. How do we approach thrombocytopenia in critically ill patients? Br J Haematol 2017; 177: 27-38.

12. Devarajan $P$. Update on mechanisms of ischemic acute kidney injury. J Am Soc Nephrol 2006; 17: 1503-20.

13. Kidney International Supplements. Kidney Disease: Improving Global Outcomes. (KDIGO) Acute Kidney Injury Work Group. KDIGO Clinical Practice Guideline for Acute Kidney Injury. Kidney Inter Suppl 2012; 2: 1-138.

14. Harrell FE Jr. Regression Modeling Strategies. NY: SpringerVerlag; 2001.

15. Kleiman NS. Are immature platelets growing up?: Toward a new marker of antiplatelet drug resistance. J Am Coll Cardiol 2016; 68: 294-6.

16. Skeith L, Baumann Kreuziger L, Crowther MA, Warkentin TE. A practical approach to evaluating postoperative thrombocytopenia. Blood Adv 2020; 4: 776-83.

17. Xu K, Chan NC, Hirsh J, et al. Quantifying immature platelets as markers of increased platelet production after coronary artery bypass grafting surgery. Eur J Haematol 2018; 101: 362-7.
18. Guthikonda S, Alviar CL, Vaduganathan M, et al. Role of reticulated platelets and platelet size heterogeneity on platelet activity after dual antiplatelet therapy with aspirin and clopidogrel in patients with stable coronary artery disease. J Am Coll Cardiol 2008; 52: 743-9.

19. Akgedik R, Yagiz Y. Is decreased mean platelet volume in allergic airway diseases associated with extent of the inflammation area? Am J Med Sci 2017; 354: 33-8.

20. Kisacik B, Tufan A, Kalyoncu U, et al. Mean platelet volume (MPV) as an inflammatory marker in ankylosing spondylitis and rheumatoid arthritis. Joint Bone Spine 2008; 75: 291-4.

21. Han JS, Park KS, Lee MJ, et al. Mean platelet volume is a prognostic factor in patients with acute kidney injury requiring continuous renal replacement therapy. J Crit Care 2014; 29: 1016-21.

22. Aydinli B, Demir A, Guclu CY, et al. Hematological predictors and clinical outcomes in cardiac surgery. J Anesth 2016; 30: 7708.

23. Ghoshal K, Bhattacharyya M. Overview of platelet physiology: its hemostatic and nonhemostatic role in disease pathogenesis. ScientificWorldJournal 2014; DOI: https://doi.org/10.1155/2014/ 781857.

24. Ittermann T, Feig MA, Petersmann A, et al. Mean platelet volume is more important than age for defining reference intervals of platelet counts. PLoS One 2019; DOI: https://doi.org/10.1371/ journal.pone. 0213658 .

25. Corash L, Chen HY, Levin J, Baker G, Lu H, Mok Y. Regulation of thrombopoiesis: effects of the degree of thrombocytopenia on megakaryocyte ploidy and platelet volume. Blood 1987; 70: 17785.

26. Kaya U, Koza Y. Predictive value of mean platelet volume in saphenous vein graft disease. Braz J Cardiovasc Surg 2018; 33: 317-22.

27. Nadim MK, Forni LG, Bihorac A, et al. Cardiac and vascular surgery-associated acute kidney injury: the $20^{\text {th }}$ International Consensus Conference of the ADQI (Acute Disease Quality Initiative) Group. J Am Heart Assoc 2018; DOI: https://doi.org/ 10.1161/JAHA.118.008834.

28. Mullens $W$, Nijst $P$. Cardiac output and renal dysfunction: definitely more than impaired flow. J Am Coll Cardiol 2016; 67: 2209-12.

29. Plicner D, Stolinski J, Wasowicz M, et al. Preoperative values of inflammatory markers predict clinical outcomes in patients after CABG, regardless of the use of cardiopulmonary bypass. Indian Heart J 2016; 68(Suppl 3): S10-5.

30. Landis RC, Brown JR, Fitzgerald D, et al. Attenuating the systemic inflammatory response to adult cardiopulmonary bypass: a critical review of the evidence base. J Extra Corpor Technol 2014; 46: 197-211.

31. Karkouti K, Wijeysundera DN, Yau TM, et al. Influence of erythrocyte transfusion on the risk of acute kidney injury after cardiac surgery differs in anemic and nonanemic patients. Anesthesiology 2011; 115: 523-30.

32. Karkouti $K$. Transfusion and risk of acute kidney injury in cardiac surgery. Br J Anaesth 2012; 109(Suppl 1): i29-38.

33. Slavka G, Perkmann T, Haslacher $H$, et al. Mean platelet volume may represent a predictive parameter for overall vascular mortality and ischemic heart disease. Arterioscler Thromb Vasc Biol 2011; 31: 1215-8.

34. Endler $G$, Klimesch A, Sunder-Plassmann H, et al. Mean platelet volume is an independent risk factor for myocardial infarction but not for coronary artery disease. Br J Haematol 2002; 117: 399404.

35. Griffin BR, Bronsert $M$, Reece TB, et al. Thrombocytopenia after cardiopulmonary bypass is associated with increased morbidity and mortality. Ann Thorac Surg 2020; 110: 50-7. 
36. Ali $U$, Gibbs R, Knight G, Tsitsikas D. Sex-divided reference intervals for mean platelet volume, platelet large cell ratio and plateletcrit using the Sysmex $\mathrm{XN}-10$ automated haematology analyzer in a UK population. Hematol Transfus Cell Ther 2019; 41: 153-7.

37. Wakeman L, Al-Ismail S, Benton A, et al. Robust, routine haematology reference ranges for healthy adults. Int $J$ Lab Hematol 2007; 29: 279-83.

38. Farias MG, Schunck EG, Dal Bo S, Martins de Castro S. Definition of reference ranges for the platelet distribution width (PDW): a local need. Clin Chem Lab Med 2010; 48: 255-7.

39. Norrasethada L, Khumpoo W, Rattarittamrong E, Rattanathammethee T, Chai-Adisaksopha C, Tantiworawit A. The use of mean platelet volume for distinguishing the causes of thrombocytopenia in adult patients. Hematol Rep 2019; DOI: https://doi.org/10.4081/hr.2019.7732
40. Bessman JD, Gilmer PR, Gardner FH. Use of mean platelet volume improves detection of platelet disorders. Blood Cells 1985; 11: 127-35.

41. Williamson DR, Albert M, Heels-Ansdell D, et al. Thrombocytopenia in critically ill patients receiving thromboprophylaxis: frequency, risk factors, and outcomes. Chest 2013; 144: 1207-15.

42. Rinder CS, Fontes M, Mathew JP, Rinder HM, Smith BR; Study of Perioperative Ischemia Research Group. Neutrophil CD11b upregulation during cardiopulmonary bypass is associated with postoperative renal injury. Ann Thorac Surg 2003; 75: 899-905.

Publisher's Note Springer Nature remains neutral with regard to jurisdictional claims in published maps and institutional affiliations. 